\title{
Estado Nutricional e Estilo de Vida de Estudantes de um Restaurante Universitário da Cidade de Salvador - BA, Brasil
}

\author{
Lisanah Rufino da Silva ${ }^{1}$ \\ Maria da Conceição Pereira da Fonseca ${ }^{1}$ \\ Carlos Rodrigo Nascimento de Lira ${ }^{1}$ \\ Bruno Santos de Assis ${ }^{1}$ \\ Celina Maria Pereira Alonso ${ }^{1}$ \\ ${ }^{1}$ Universidade Federal da Bahia, UFBA, Brasil; E-Mails: mcfonsec@ufba.br; mcfonseca@gmail.com; \\ celinaalonso@terra.com.br
}

\section{Resumo}

Com objetivo conhecer o Estado Nutricional - EN e o estilo de vida dos estudantes bolsistas de um restaurante universitário, localizado em Salvador-Ba, Brasil foi realizado com 70 universitários, maiores de 18 anos, em 2016 um estudo de corte transversal através da aplicação de questionário estruturado, com informações socioeconômicas, estilo de vida e atividade física. Avaliou-se também o EN, com cálculo de Índice de Massa Corporal- IMC, Circunferência da Cintura - CC e percentual de gordura - \%GC, usando-se para esses os critérios de classificação proposto pela Organização Mundial da Saúde, com a verificação de diferenças significativas entre os sexos, pelo teste de Fisher. A maioria dos entrevistados era de adultos jovens com massa corporal na faixa de normalidade, mas com presença de sobrepeso e obesidade em parcela deles, atrelada ao consumo de álcool, de tabaco e hábitos alimentares inadequados, sendo fatores importantes na gênese de Doenças Crônicas Não Transmissíveis - DCNT.

Palavras-chave: alimentação coletiva; estado nutricional; estudantes universitários; hábitos alimentares.

\begin{abstract}
In order to know the Nutritional Status - NS and the lifestyle of the students of a university restaurant, located in Salvador-Bahia, Brazil, was carried out with 70 university students, over 18 years of age, in 2016 a cross-sectional study through the application of Structured questionnaire with socioeconomic information, lifestyle and physical activity. It was also evaluated the EN, with calculation of Body Mass Index-BMI, Waist Circumference - WC and percentage of fat $-\% \mathrm{BF}$, using the classification criteria proposed by the World Health Organization for verification of Significant differences by the Fisher test, between the sexes. The majority of the interviewees were young adults with normal body mass, but with overweight and obesity in a portion of them, linked to alcohol consumption, tobacco consumption and inadequate eating habits, being important factors in the genesis of Chronic Noncommunicable Diseases - NCDs.
\end{abstract}

Keywords: collective feeding; eating habits; nutritional status; university students.

\section{Introdução}

$\mathrm{O}$ ato de comer consiste muito além da ingestão de nutrientes para o bom funcionamento do organismo. A alimentação é uma necessidade básica, um direito humano, que não está restrita apenas às questões biológicas, mas se relaciona diretamente com questões psicológicas, econômicas e sociais (Batista Filho, 1999; Garcia, 1993). A nutrição é fundamental para a saúde em todas as fases da vida, desta forma deficiências ou excessos alimentares podem contribuir para inúmeros problemas de saúde e baixa qualidade de vida (São Paulo, 2010). 
Ao ingressar no ambiente universitário são estabelecidas novas relações sociais, que podem levar os jovens as mudanças comportamentais e adoção de práticas que causem risco à sua saúde. As alterações ocorrem principalmente no padrão alimentar favorecendo o desequilíbrio na ingestão de nutrientes, no consumo de álcool e tabagismo, e atrelado a isso se observa redução do nível de atividade física (Costa \& Vasconcelos 2010; Franca \& Colares, 2008).

Este padrão ou práticas de riscos observadas nos universitários são reflexos da tendência mundial e um dos principais fatores desencadeadores da transição nutricional, que se caracteriza por decréscimo no número de pessoas com desnutrição e aumento nos níveis de sobrepeso e obesidade na população (Monteiro, Mondini, \& Costa, 2000). No Brasil, uma forte característica de mudança no padrão alimentar da população foi de aumento da dieta rica em gorduras, açúcares refinados, alimentos refinados e redução em carboidratos complexos e fibras, e reduzida ingestão de frutas, hortaliças e fotoquímicos, sendo estas características marcantes do padrão alimentar dos universitários (Monteiro, Mondini, \& Costa, 2000) ${ }^{1}$.

O estilo de vida universitário e o padrão alimentar estão associados ao advento de Doenças Crônicas Não Transmissíveis (DCNT), uma vez que é comum entre os estudantes a omissão de refeições e a preferência por àquelas nutricionalmente desequilibradas.

A associação da dieta de alta densidade energética, o sedentarismo, consumo frequente de bebida alcoólica e o tabagismos podem contribuir para a incidência de sobrepeso e obesidade nessa população, fator este determinante para o surgimento de diabetes mellitus tipo 2, dislipidemias e hipertensão, além de aumentar o risco de certos tipos de câncer (WHO, 2002).

Vale destacar que o hábito etilista e o tabagismos são fatores que interferem na qualidade de vida, como também influenciam negativamente o Estado Nutricional (EN) de indivíduos (Kachani, Brasiliano, \& Hochgraf, 2008). No Brasil pesquisas sugerem que o ingresso na universidade deixa os jovens com maior vulnerabilidade para o início e a manutenção do uso de álcool e outras drogas (Peuker, Fogaça, \& Bizarro, 2006).

A literatura já tem demostrado importante prevalência de sobrepeso/obesidade entre os estudantes de universidades brasileiras $^{2}$. As refeições consumidas diariamente fora de casa, não podem ser consideradas isoladamente como única responsável pelo estado nutricional, pois dependem também do estilo de vida que é empregado, entretanto estudos evidenciam que à alimentação fora do lar podem ter uma pior qualidade devido a maior densidade energética, podendo contribuir para o excesso de peso, demonstrando que essas refeições podem exercer efeito negativo para a saúde dos comensais, com isso evidencia a importância de promover a alimentação saudável nesses estabelecimentos ${ }^{3}$.

Destaca-se que desde 2010 no Brasil, as matrículas de graduação vêm aumentando, apresentando um acréscimo de 5,6\% no número de ingressantes nas Universidades do país, contribuindo para atingir um total de 7.305.977 estudantes no ano de $2013^{4}$. Portanto, torna-se importante a vigilância do estado nutricional dos universitários no Brasil, dado o crescente número destes e os diversos fatores que propiciam ao aumento de sobrepeso e obesidade neste estrato da sociedade. Vale destacar que uma das formas de monitorar o estado de saúde da população é pela avaliação do estado nutricional que tem entre os seus principais objetivos a de estimar a magnitude e distribuição dos problemas nutricionais e a de identificar fatores que possam estar interferindo direta ou indiretamente para a situação observada, além de sugerir medidas capazes de melhorar a saúde desta população (São Paulo, 2010).

Portanto, este estudo tem o objetivo conhecer e descrever o estado nutricional e o estilo de vida dos estudantes bolsistas de um Restaurante Universitário (RU) da cidade de Salvador-Ba, tendo em vista também o crescimento no número de estudantes bolsistas e realização de suas principais refeições no restaurante universitário e além da carência de estudos sobre a participação da dieta consumida diariamente nesses locais.

\footnotetext{
${ }^{1}$ Trabalho realizado com o objetivo de analisar características da alimentação e da atividade física em universitários de uma instituição privada.

${ }^{2}$ Estudo realizado com estudantes da Universidade do Planalto Catarinense com objetivo de verificar os níveis de atividade física, hábitos alimentares e controle de peso corporal em universitários.

${ }^{3}$ Qualidade das refeições oferecidas por empresas cadastradas pelo Programa de Alimentação do Trabalhador na cidade de Santos-SP. Objetivo do estudo foi avaliar a qualidade global das refeições oferecidas no PAT na cidade de Santos-SP, Brasil. Para avaliar a qualidade global das refeições oferecidas por quatro empresas durante três dias consecutivos.

${ }^{4}$ Link disponível em: <http://download.inep.gov.br/download/superior/censo/2013/resumo_tecnico_censo_educacao_superior_2013.pdf>

Revista Internacional em Língua Portuguesa, 2018, №33, Ciências da Saúde e Tecnologia, pp. 131-146 


\section{Métodos}

Trata-se de um estudo de corte transversal, com coleta em outubro e novembro de 2016, desenvolvido em um RU da cidade de Salvador - Ba. O protocolo desse estudo foi submetido ao Comitê de Ética e Pesquisa da Escola de Nutrição da Universidade Federal da Bahia - UFBA e aprovado por parecer do processo no 228.318/2012.

A amostra foi definida após levantamento realizado por estudo piloto, tendo como objetivo testar a aplicabilidade do questionário, verificar a prevalência de alguns desfechos, treinar questões logísticas, e, sobretudo levantar dados para uma variável quantitativa contínua, no caso Índice de Massa Corporal (IMC), para determinar a média ( $\mu$ ) e o desvio padrão $(\sigma)$, utilizados $n$ fórmula para a determinação do tamanho mínimo da amostra. Com isso, obteve a média ( $\mu$ ) e desvio padrão $(\sigma)$ do IMC, tendo sido obtido confiança de $95 \%$, com erro de $22 \%$ do desvio padrão e com IMC de 24,12 a 26,21 .

Participaram do estudo 70 universitários de ambos os sexos, sorteados aleatoriamente. Os critérios de inclusão foram: idade $\geq 18$ anos, receber gratuidade para alimentação da Pró-Reitoria de Assistência Estudantil (PROAE) da Universidade e realizar no mínimo três refeições por semana no RU. Os participantes foram informados do objetivo do estudo, e aqueles que concordaram foram convidados a ler e assinar o Termo de Consentimento Livre e Esclarecido (TCLE).

A coleta dos dados foi realizada pela aplicação de questionário estruturado para coleta das variáveis socioeconômicas, possíveis fatores de risco para DCNT e declaração dos estudantes quanto ao consumo de bebidas alcoólicas e hábitotabagista. Efetuado por oito estudantes do curso de Nutrição de uma Universidade Pública da cidade de Salvador-Ba, os mesmos foram devidamente treinados com supervisão dos nutricionistas do Núcleo de Segurança Alimentar (NUSA) da Universidade. O instrumento utilizado para medida do Nível De Atividade Física (NAF) foi adaptado do Questionário Internacional de Atividade Física (IPAQ) validado por Matsudo et al. (2001) ${ }^{5}$. Os dados foram classificados de acordo com a orientação do IPAQ nas categorias em: sedentário, irregularmente ativo (A e B), ativo, muito ativo.

Para avaliar o padrão alimentar, foi aplicado o questionário validado "Como está sua alimentação?" elaborado pela Coordenação Geral de Alimentação e Nutrição (CGAN) do Ministério da Saúde ${ }^{6}$. Após responder o questionário, foi feito um somatório das alternativas assinaladas pelos participantes, onde de acordo com a pontuação calculada a qualidade da alimentação foi classificada em três categorias: ruim para pontuação até 28, regular para pontuação de 29 a 42 e boa para pontuação acima de 42 pontos.

A coleta de dados antropométricos consistiu na mensuração do peso, altura, Circunferência da Cintura (CC) e as quatro pregas cutâneas (bicipital, tricipital, subescapular e suprailíaca). Utilizou-se a técnica padronizada por Lohman e colaboradores, (Lohman, Roche, \& Martorell, 1988). Todas as medidas foram realizadas duas vezes pelo mesmo examinador para cada pessoa, calculando-se ao final a média aritmética das mesmas. Nos casos em que a diferença foi maior que $100 \mathrm{~g}$ para peso e de $0,2 \mathrm{~cm}$ para altura, CC e 0,2 $\mathrm{mm}$ para as pregas cutâneas as medidas foram repetidas.

Para aferir o peso utilizou-se como instrumento uma balança digital (da marca Plenna, modelo MEA 07400, com capacidade de $150 \mathrm{~kg}$ ). A altura foi aferida utilizando um estadiômetro (da marca SECA, modelo E0123) fixado a 2,20 metros verticais em relação ao piso, em parede sem rodapé. O IMC foi obtido pela razão entre peso por altura ao quadrado e sua classificação seguiu recomendações da Organização Mundial de Saúde (OMS) (OMS, 2000), onde desnutridos apresentam IMC $<18,5 \mathrm{~kg} / \mathrm{m}^{2}$, eutrófico IMC entre 18,5 kg/m² -24,99 kg/m², sobrepeso IMC entre 25 $\mathrm{kg} / \mathrm{m}^{2}-29,99 \mathrm{~kg} / \mathrm{m}^{2}$ e obesidade IMC $\geq 30 \mathrm{~kg} / \mathrm{m}^{2}$.

Os valores da CC foram comparados com os valores de referência para risco de complicações metabólicas associadas à obesidade, segundo as recomendações da OMS (2002), onde homens com CC $\geq 94 \mathrm{~cm}$ são considerados com risco elevado e $\geq 102 \mathrm{~cm}$, muito elevado e mulheres $\geq 80 \mathrm{~cm}$, como elevado e $\geq 88 \mathrm{~cm}$ muito elevado.

A avaliação do percentual de gordura foi obtida por meio do somatório de pregas cutâneas coletadas obedecendo às técnicas de medida descrita por Lohman e colaboladores (1988) com auxílio de um adipômetro da marca Skinfold

\footnotetext{
${ }^{5}$ Este estudo é parte de um esforço internacional para validar um Questionário Internacional de Atividade Física (IPAQ) proposto pela Organização Mundial de Saúde (1998).

${ }^{6}$ Link disponível em: <http://nutricao.saude.gov.br/teste_alimentacao.php> 
Caliper-Saehan, modelo SH5020 e calibrado. Utilizou-se a referência descrita por Durnin e Womersley $(1974)^{7}$ na sua classificação.

Para análise dos dados foram calculadas as frequências simples e absoluta; médias, desvio padrão, bem como foi realizada a avaliação das diferenças significativas dos dados obtidos entre os homens e mulheres, utilizando o teste exato de Fisher, com um nível de significância de 0,05.

\section{Resultados}

Foram avaliados 70 indivíduos com idades entre 18 e 56 anos e com renda familiar mensal de 1 a 2 salários mínimos. Em relação ao estilo de vida, foi observado que $67,14 \%$ dos estudantes entrevistados eram etilistas e $7,14 \%$ tabagistas. No que tange a presença de história familiar de DCNT, observou-se um percentual de 55,71\% dos estudantes apresentavam história familiar para Hipertensão Arterial Sistêmica (HAS), 48,57\% para Diabetes Mellitus (DM), seguido de Câncer (34,29\%), obesidade (27,14\%) e Doenças Cardiovasculares (DCV) (21,43\%). Observou-se baixa prevalência de história familiar para doença renal (14,28\%) (Tabela 1).

Tabela 1. Perfil demográfico, socioeconômico, estilo de vida e histórico de doenças na família dos bolsistas da PROAE frequentadores de um RU de Salvador - Ba, Brasil, 2016.

\begin{tabular}{|c|c|c|c|c|c|c|}
\hline Variáveis & \multicolumn{2}{|c|}{ Masculino } & \multicolumn{2}{|c|}{ Feminino } & \multicolumn{2}{|c|}{ Total } \\
\hline Faixa Etária & $\mathrm{N}$ & $\%$ & $\mathrm{~N}$ & $\%$ & $\mathrm{~N}$ & $\%$ \\
\hline$<20$ & 2 & 2,86 & 0 & 0,00 & 2 & 2,86 \\
\hline 20 a 35 & 31 & 44,29 & 29 & 41,43 & 60 & 85,71 \\
\hline 36 a 56 & 5 & 7,14 & 3 & 4,29 & 5 & 11,43 \\
\hline Total & 38 & 54,29 & 32 & 45,71 & 70 & 100 \\
\hline \multicolumn{7}{|c|}{ Renda Familiar } \\
\hline$<1 \mathrm{SM}$ & 15 & 21,43 & 14 & 20,00 & 29 & 41,43 \\
\hline 1 a 2 SM & 20 & 28,57 & 16 & 22,86 & 36 & 51,43 \\
\hline$<2 \mathrm{SM}$ & 3 & 4,29 & 2 & 2,86 & 5 & 7,14 \\
\hline Total & 38 & 54,29 & 32 & 45,71 & 70 & 100 \\
\hline \multicolumn{7}{|c|}{ Consumo de Álcool } \\
\hline Sim & 28 & 40,00 & 19 & 27,14 & 47 & 67,14 \\
\hline Não & 10 & 14,29 & 13 & 18,57 & 23 & 32,86 \\
\hline Total & 38 & 54,29 & 32 & 45,71 & 70 & 100 \\
\hline \multicolumn{7}{|c|}{ Hábito de Fumar } \\
\hline Sim & 4 & 5,71 & 1 & 1,43 & 5 & 7,14 \\
\hline Não & 34 & 48,57 & 31 & 44,29 & 65 & 92,86 \\
\hline Total & 38 & 54,29 & 32 & 45,71 & 70 & 100 \\
\hline \multicolumn{7}{|c|}{ Histórico de Doenças na Família } \\
\hline $\begin{array}{l}\text { Hipertensão Arterial } \\
\text { Sistêmica }\end{array}$ & 18 & 25,71 & 21 & 30,00 & 39 & 55,71 \\
\hline Diabetes Mellitus & 14 & 20,00 & 20 & 28,57 & 34 & 48,57 \\
\hline Doença Renal & 6 & 8,57 & 4 & 5,71 & 10 & 14,29 \\
\hline Doença Cardiovascular & 6 & 8,57 & 9 & 12,86 & 15 & 21,43 \\
\hline Obesidade & 6 & 8,57 & 9 & 12,86 & 19 & 27,14 \\
\hline Câncer & 14 & 20,00 & 10 & 14,29 & 24 & 34,29 \\
\hline
\end{tabular}

Notas: Coleta de Campo deste estudo, 2016.

A população em estudo foi composta de adultos jovens, com média de idade próxima de 26 e 27 anos, sendo que a maioria (85,71\%) estava entre a faixa etária de 20 a 35 anos, com peso adequado para a altura e sem risco para desenvolver doenças metabólicas, conforme IMC médio de 23,68 (Tabelas 1 e 2). No entanto observa-se um percentual de 32,86\% de sobrepeso e obesidade, segundo IMC (Tabela 2).

\footnotetext{
7 Disponível em: <https://www.cambridge.org/core/services/aop-cambridge-core/content/view/DAC-8BA25856FCEB30E22F60E0AF80D07/S0 007114574000614a.pdf/body_fat_assessed_from_total_body_density_and_its_estimation_from_skinfold_thickness_measurements_on_481_men _and_women_aged_from_16_to_72_years.pdf> 
Tabela 2. Dados antropométricos e estado nutricional dos bolsistas da PROAE frequentadores de um RU de Salvador Ba, Brasil, 2016.

\begin{tabular}{|c|c|c|c|c|c|c|}
\hline \multirow{2}{*}{$\begin{array}{l}\text { Variáveis } \\
\text { População } \\
\text { estudada }\end{array}$} & \multicolumn{2}{|c|}{ Masculino } & \multicolumn{2}{|c|}{ Feminino } & \multicolumn{2}{|c|}{ População Total } \\
\hline & Média & Desvio Padrão & Média & Desvio Padrão & Média & $\begin{array}{l}\text { Desvio } \\
\text { Padrão }\end{array}$ \\
\hline $\begin{array}{l}\text { Idade (anos) } \\
\text { média }\end{array}$ & 27,37 & 7,30 & 25,75 & 5,75 & 26,63 & \\
\hline Peso (Kg) média & $73,29 a$ & 10,05 & $61,93 b$ & 13,33 & 68,09 & \\
\hline $\begin{array}{l}\text { Altura }(\mathrm{m}) \\
\text { média }\end{array}$ & $1,76 a$ & 0,06 & $1,6 \mathrm{a}$ & 0,08 & 1,69 & \\
\hline $\begin{array}{l}\mathrm{IMC}\left(\mathrm{Kg} / \mathrm{m}^{2}\right) \\
\text { médio }\end{array}$ & $23,64 a$ & 3,40 & $23,72 b$ & 4,50 & 23,68 & \\
\hline $\mathrm{CC}(\mathrm{cm})$ Médio & $80,11 a$ & 8,49 & $74,56 b$ & 9,38 & 77,58 & \\
\hline$\% G C$ média & $21,59 a$ & 4,24 & $34,9 b$ & 3,86 & 27,68 & \\
\hline IMC (OMS) & $\mathbf{N}$ & $\%$ & $\mathbf{N}$ & $\%$ & $\mathbf{N}$ & $\%$ \\
\hline Baixo Peso & 4 & 10,53 & 2 & 6,25 & 6 & 8,57 \\
\hline Eutrofia & 20 & 52,63 & 21 & 65,63 & 41 & 58,57 \\
\hline $\begin{array}{c}\text { Sobrepeso e } \\
\text { obesidade }\end{array}$ & 14 & 36,84 & 9 & 28,13 & 23 & 32,86 \\
\hline Total & 38 & 100 & 32 & 100 & 70 & 100 \\
\hline $\begin{array}{c}\text { Circunferência } \\
\text { da Cintura }\end{array}$ & $\mathbf{N}$ & $\%$ & $\mathbf{N}$ & $\%$ & $\mathbf{N}$ & $\%$ \\
\hline $\begin{array}{l}\mathrm{CC}(\mathrm{cm}) \mathrm{Sem} \\
\text { risco }\end{array}$ & 36 & 94,74 & 23 & 71,88 & 59 & 84,29 \\
\hline $\begin{array}{l}\text { CC (cm) Risco } \\
\text { Aumentado }\end{array}$ & 2 & 5,26 & 9 & 28,13 & 11 & 15,72 \\
\hline Total & 38 & 100 & 32 & 100 & 70 & 100 \\
\hline \% GC (OMS) & $\mathbf{N}$ & $\%$ & $\mathbf{N}$ & $\%$ & $\mathbf{N}$ & $\%$ \\
\hline \%GC Desejável & 4 & 10,53 & 0 & 0,00 & 4 & 5,71 \\
\hline \%GC Limítrofe & 27 & 71,05 & 6 & 18,75 & 33 & 47,14 \\
\hline \%GC Elevado & 7 & 18,42 & 26 & 81,25 & 33 & 47,14 \\
\hline Total & 38 & 100 & 32 & 100 & 70 & 100 \\
\hline
\end{tabular}

Fonte: Coleta de Campo deste estudo, 2016.

Letras diferentes $(a, b)$ há diferença estatisticamente Significativas pelo teste Exato de Fisher; Letras iguais ( $a$, a) não há diferença estatisticamente Significativas pelo teste Exato de Fisher.

Observou-se que o percentual de estudantes que estavam com CC normal foi $84,29 \%$ e aqueles que estavam com CC em risco aumentado ou muito aumentado para doenças metabólicas na população total foi de $15,71 \%$. Já para os estudantes que apresentaram CC elevada e muito elevada de acordo com o sexo, observa-se que no público feminino o percentual foi maior $28,13 \%$, comparado com público masculino que apresentou 5,26\%. Quanto ao Percentual de Gordura Corporal (\%GC) total, $47,14 \%$ estavam na faixa de risco à obesidade, sendo que a maioria era do sexo feminino (81,25\%), já o percentual para aqueles que estavam com \%GC desejável e limítrofe foi 52,85\% (Tabela 2).

Cabe mencionar que foi realizado analise de diferenças significativas para os valores médios obtidos de peso, altura, IMC, CC e \%CG entre homens e mulheres, sendo que destes destaca-se as diferenças significativas observadas entre os homens e mulheres para CC e \%CG médio (Tabela 2).

Tabela 3. Relação do estado nutricional, segundo IMC e percentual de gordura corporal dos bolsistas da PROAE frequentadores de um RU de Salvador - Ba, Brasil, 2016.

\begin{tabular}{|c|c|c|c|c|c|c|c|}
\hline \multirow{3}{*}{$\begin{array}{c}\text { Estado } \\
\text { Nutricional }\end{array}$} & \multirow{3}{*}{ Sexo } & \multicolumn{2}{|c|}{ Total } & \multicolumn{4}{|c|}{ Percentual de Gordura Corporal (\%GC) } \\
\hline & & & & \multicolumn{2}{|c|}{ Normal } & \multicolumn{2}{|c|}{ Elevado } \\
\hline & & $\mathrm{N}$ & $\%$ & $\mathrm{~N}$ & $\%$ & $\mathrm{~N}$ & $\%$ \\
\hline \multirow{3}{*}{ Baixo Peso } & $\mathrm{F}$ & 2 & 2,86 & 2 & 2,86 & 0 & 0 \\
\hline & $\mathrm{M}$ & 4 & 5,71 & 4 & 5,71 & 0 & 0 \\
\hline & $\mathrm{T}$ & 6 & 8,57 & 6 & 8,57 & 0 & 0 \\
\hline \multirow{3}{*}{ Eutrofia } & $\mathrm{F}$ & 21 & 30,00 & 4 & 5,71 & 17 & 24,29 \\
\hline & $\mathrm{M}$ & 20 & 28,57 & 19 & 27,14 & 1 & 1,43 \\
\hline & $T$ & 41 & 58,57 & 23 & 32,86 & 18 & 25,71 \\
\hline
\end{tabular}




\begin{tabular}{|c|c|c|c|c|c|c|c|}
\hline \multirow{3}{*}{$\begin{array}{c}\text { Sobrepeso } \\
\text { e }\end{array}$} & $\mathrm{F}$ & 9 & 12,86 & 0 & 0,00 & 9 & 12,86 \\
\cline { 2 - 8 } Obesidade & $\mathrm{M}$ & 14 & 20,00 & 8 & 11,43 & 6 & 8,57 \\
\cline { 2 - 8 } & $\mathrm{T}$ & 23 & 32,86 & 8 & 11,43 & 15 & 21,43 \\
\hline \multirow{3}{*}{ Total } & $\mathrm{F}$ & 32 & 45,71 & 6 & 8,57 & 26 & 37,14 \\
\cline { 2 - 8 } & $\mathrm{M}$ & 38 & 54,29 & 31 & 44,29 & 7 & 10,00 \\
\cline { 2 - 8 } & $\mathrm{T}$ & 70 & 100 & 37 & 52,86 & 33 & 47,14 \\
\hline
\end{tabular}

Fonte: Coleta de Campo deste estudo, 2016.

No que diz respeito a associação do EN, segundo IMC, relacionado às pregas cutâneas observou-se que todos os estudantes com baixo peso apresentaram frequência de \%GC normal. Entre aqueles eutróficos à maioria estava dentro da normalidade (32,85\%). Contudo é observado que cerca $26 \%$ encontravam-se com risco a obesidade. Quanto ao percentual de gordura corporal elevado, pode-se observar que $47,14 \%$ um sendo $37,14 \%$ mulheres e $10,00 \%$ homens (Tabela 3). Observou-se que 14,29\% dos estudantes apresentaram CC em risco para doenças metabólicas em ambos os sexos, sendo $11,43 \%$ do sexo feminino e $2,86 \%$ do sexo masculino (Tabela 4 ).

Tabela 4. Relação do estado nutricional de Sobrepeso e Obesidade, segundo IMC e Circunferência da Cintura (CC) dos bolsistas da PROAE frequentadores de um RU de Salvador - Ba, Brasil, 2016.

\begin{tabular}{|c|c|c|c|c|c|c|c|}
\hline \multirow{2}{*}{$\begin{array}{c}\text { Estado } \\
\text { Nutricional }\end{array}$} & \multirow{2}{*}{ Sexo } & \multicolumn{2}{|c|}{ Total } & \multicolumn{4}{c|}{ Circunferência da Cintura (CC) } \\
\cline { 3 - 8 } & & $\mathrm{N}$ & $\%$ & $\mathrm{~N}$ & $\%$ & $\mathrm{~N}$ & $\%$ \\
\hline $\begin{array}{c}\text { Sobrepeso } \\
\mathrm{e}\end{array}$ & $\mathrm{F}$ & 9 & 12,86 & 5 & 7,14 & 3 & 4,29 \\
\cline { 2 - 8 } \\
\cline { 2 - 8 } \\
\cline { 2 - 8 }
\end{tabular}

Fonte: Coleta de Campo deste estudo, 2016.

Em relação ao estilo de vida, verifica-se que atividade física dos estudantes com peso adequado para altura ( $\mathrm{n}=41$ ) foi classificada em sua maioria como insuficiente ativo (A e B), estes apresentaram percentual de 25,71\%, já os entrevistados sedentários apresentaram menor frequência $(n=6)$ quando comparado ao insuficiente ativo $A$ e $B$ $(n=11)$. (Tabela 5). No que diz respeito ao hábito alimentar e EN, verifica-se que a maior parte dos universitários foi classificada com o hábito alimentar regular (75,71\%) e 6\% apresentaram hábito alimentar e EN classificados como ruim (Tabela 6).

Tabela 5. Perfil nutricional, segundo IMC de acordo com a Atividade Física dos bolsistas da PROAE, frequentadores de um RU de Salvador, Ba, 2016.

\begin{tabular}{|c|c|c|c|c|c|c|c|c|c|}
\hline \multirow{3}{*}{$\begin{array}{l}\text { Atividade } \\
\text { Física }\end{array}$} & \multirow{3}{*}{ Sexo } & \multicolumn{6}{|c|}{ Estado nutricional } & \multirow{2}{*}{\multicolumn{2}{|c|}{ Total }} \\
\hline & & \multicolumn{2}{|c|}{ Baixo Peso } & \multicolumn{2}{|c|}{ Eutrofia } & \multicolumn{2}{|c|}{$\begin{array}{c}\text { Sobrepeso e } \\
\text { Obesidade }\end{array}$} & & \\
\hline & & $\mathrm{N}$ & $\%$ & $\mathrm{~N}$ & $\%$ & $\mathrm{~N}$ & $\%$ & $\mathrm{~N}$ & $\%$ \\
\hline \multirow{3}{*}{ Sedentário } & $\mathrm{F}$ & 1 & 1,43 & 4 & 5,71 & 5 & 7,14 & 10 & 14,29 \\
\hline & $M$ & 1 & 1,43 & 2 & 2,86 & 1 & 1,43 & 4 & 5,71 \\
\hline & $T$ & 2 & 2,86 & 6 & 8,57 & 6 & 8,57 & 14 & 20,00 \\
\hline \multirow{3}{*}{$\begin{array}{l}\text { Irregularmente } \\
\text { Ativo A }\end{array}$} & $\mathrm{F}$ & 1 & 1,43 & 4 & 5,71 & 1 & 1,43 & 6 & 8,57 \\
\hline & $M$ & 2 & 2,86 & 3 & 4,29 & 1 & 1,43 & 6 & 8,57 \\
\hline & $T$ & 3 & 4,29 & 7 & 10,00 & 2 & 2,86 & 12 & 17,14 \\
\hline \multirow{3}{*}{$\begin{array}{c}\text { Irregularmente } \\
\text { Ativo B }\end{array}$} & $\mathrm{F}$ & 0 & 0,00 & 7 & 10,00 & 0 & 0,00 & 7 & 10,00 \\
\hline & $M$ & 1 & 1,43 & 4 & 5,71 & 1 & 1,43 & 6 & 8,57 \\
\hline & $T$ & 1 & 1,43 & 11 & 15,71 & 1 & 1,43 & 13 & 18,57 \\
\hline \multirow{3}{*}{ Ativo } & $\mathrm{F}$ & 0 & 0,00 & 1 & 1,43 & 1 & 1,43 & 2 & 2,86 \\
\hline & $M$ & 0 & 0,00 & 9 & 12,86 & 8 & 11,43 & 17 & 24,29 \\
\hline & $T$ & 0 & 0,00 & 10 & 14,29 & 9 & 12,86 & 19 & 27,14 \\
\hline \multirow{3}{*}{ Muito Ativo } & $\mathrm{F}$ & 0 & 0,00 & 5 & 7,14 & 2 & 2,86 & 7 & 10,00 \\
\hline & $M$ & 0 & 0,00 & 2 & 2,86 & 3 & 4,29 & 5 & 7,14 \\
\hline & $T$ & 0 & 0,00 & 7 & 10,00 & 5 & 7,14 & 12 & 17,14 \\
\hline
\end{tabular}

Fonte: Coleta de Campo deste estudo, 2016. 
Tabela 6. Perfil nutricional, segundo IMC e hábito alimentar dos bolsistas da PROAE frequentadores de um RU de Salvador, Ba, 2016

\begin{tabular}{|c|c|c|c|c|c|c|c|c|c|}
\hline \multirow{3}{*}{$\begin{array}{c}\text { Estado } \\
\text { Nutricional }\end{array}$} & \multirow{3}{*}{ Sexo } & \multicolumn{6}{|c|}{ Estado Nutricional } & \multirow{2}{*}{\multicolumn{2}{|c|}{ Total }} \\
\hline & & \multicolumn{2}{|c|}{ Baixo Peso } & \multicolumn{2}{|c|}{ Eutrofia } & \multicolumn{2}{|c|}{$\begin{array}{l}\text { Sobrepeso e } \\
\text { Obesidade }\end{array}$} & & \\
\hline & & $\mathrm{N}$ & $\%$ & $\mathrm{~N}$ & $\%$ & $\mathrm{~N}$ & $\%$ & $\mathrm{~N}$ & $\%$ \\
\hline \multirow{3}{*}{ Ruim } & $\mathrm{F}$ & 0 & 0,00 & 0 & 0,00 & 0 & 0,00 & 0 & 0,00 \\
\hline & $\mathrm{M}$ & 0 & 0,00 & 2 & 2,86 & 2 & 2,86 & 4 & 5,71 \\
\hline & Total & 0 & 0,00 & & 2,86 & 2 & 2,86 & 4 & 5,71 \\
\hline \multirow{3}{*}{ Regular } & $\mathrm{F}$ & 1 & 1,43 & 18 & 25,71 & 5 & 7,14 & 24 & 34,29 \\
\hline & $\mathrm{M}$ & 4 & 5,71 & 17 & 24,29 & 8 & 11,43 & 29 & 41,43 \\
\hline & Total & 5 & 7,14 & 35 & 50,00 & 13 & 18,57 & 53 & 75,71 \\
\hline \multirow{3}{*}{ Bom } & $\mathrm{F}$ & 1 & 1,43 & 3 & 4,29 & 4 & 5,71 & 8 & 11,43 \\
\hline & $\mathrm{M}$ & 0 & 0,00 & 1 & 1,43 & 4 & 5,71 & 5 & 7,14 \\
\hline & Total & 1 & 1,43 & 4 & 5,71 & 8 & 11,43 & 13 & 18,57 \\
\hline \multirow{3}{*}{ Total } & $\mathrm{F}$ & 2 & 2,86 & 21 & 30,00 & 9 & 12,86 & 32 & 45,71 \\
\hline & $\mathrm{M}$ & 4 & 5,71 & 20 & 28,57 & 14 & 20,00 & 38 & 54,29 \\
\hline & Total & 6 & 8,57 & 39 & 58,57 & 23 & 32,86 & 70 & 100 \\
\hline
\end{tabular}

Fonte: Coleta de Campo deste estudo, 2016.

\section{Discussão}

O estado nutricional antropométrico, hábito alimentar e estilo de vida, constituem fatores que influenciam sensivelmente no estado geral de saúde de jovens universitários. No entanto são escassos os estudos que se propõem a estudar esses fatores, principalmente em estudantes de baixa condição econômica e social.

Em se tratando de jovens universitários, vale considerar que fatores como o desligamento da casa dos pais e a influencia na aquisição de novas responsabilidades, já que esses estudantes passam a ter mais autonomia sobre sua vida são fatores que podem influenciar em diferentes aspectos, sobretudo no âmbito alimentar. Com a rotina intensa e associada ao baixo poder de compra, estudantes tendem a omitir refeições, dando preferência pelo consumo de lanches rápidos e combinações alimentares nutricionalmente desequilibradas (Tardio \& Falcão, 2006). Esse padrão alimentar se correlaciona diretamente com alterações no controle de peso, sendo importante fator ambiental para a gênese do sobrepeso e obesidade.

A Organização Mundial de Saúde (OMS) apresenta a obesidade como um dos maiores problemas de saúde pública no mundo. A projeção é que, em 2025, cerca de 2,3 bilhões de adultos estejam com sobrepeso; e mais de 700 milhões, obesos. No Brasil, a obesidade vem crescendo cada vez mais. Alguns estudos mostram que mais de 50\% da população está acima do peso, ou seja, na faixa de sobrepeso e obesidade (ABESO, 2010). O estilo de vida moderno favorece o ganho de peso por diversos fatores que interferem na ingestão alimentar, a necessidade de se realizar refeições em curto espaço de tempo atrapalha os mecanismos de saciedade, contribuindo para o excesso de peso e desenvolvimento de outras DCNT (ABESO, 2016).

Embora a maioria da população estudada esteja com peso adequado para altura, é visto que uma parcela considerável $(32,86 \%)$ apresenta sobrepeso e obesidade, confirmando uma tendência comum já que nas últimas décadas, as prevalências de sobrepeso e obesidade em populações adultas vêm crescendo não só em países desenvolvidos como também nos países em desenvolvimento. Tal fato é verificado em estudos com universitários atribuindo ao excesso de gordura corporal proporções epidêmicas (WHO, 2007) $)^{8}$.

Diante do exposto, o aumento no número de sobrepeso e obesidade, fomenta a implementação de estratégias de promoção de saúde, direcionadas para os estudantes universitários do presente estudo, tendo em vista que os fatores que podem influenciar fortemente no EN são o consumo alimentar atrelado ao sedentarismo ou com níveis de atividade física irregular.

\footnotetext{
${ }^{8}$ Prevalencia de obesidad e hipercolesterolemia en la Facultad de Medicina de la Universidad Peruana Cayetano Heredia - 1998, disponível em: <http://www.upch.edu.pe/vrinve/dugic/revistas/index.php/RMH/article/view/2388/2338>.
} 
O maior risco para doenças metabólicas foi encontrado no grupo feminino quando comparado ao masculino (11,43\% tanto de risco aumentado, quanto risco muito aumentado), diferindo do estudo de Staudt e Mattos (2011) ${ }^{9}$, onde mostra que o sexo que apresentou maior risco de doenças cardiovasculares de acordo com a CC foi o masculino. Rezende et al. (2006) avaliando o perfil antropométrico e de composição corporal em ambos os sexos segundo as categorias de IMC, verificou que as mulheres apresentaram valores porcentuais de gordura corporal maiores que os dos homens em todas as categorias do IMC, observou ainda que na categoria de sobrepeso em ambos os sexos dos indivíduos já apresentavam medida de CC elevada. Considerando essa tendência crescente do sobrepeso e obesidade na população brasileira em geral e a sua associação com DCNT, fazem-se necessárias intervenções visando reduzir o peso corporal, em especial a gordura central na região abdominal, sendo condutas de relevante importância para a prevenção e controle das DCNT na população.

Em relação a presença de história familiar de DCNT, como a HAS e o DM, sabe-se que tais doenças figuram como principal causa de mortalidade e incapacidade no mundo, responsável por $59 \%$ dos 56,5 milhões de óbitos anuais (Guiesse \& Lopes, 2013). No estudo de Petribú $(2009)^{10}$, realizado com universitários de Pernambuco, foi observada uma proporção de hipertensão, diabetes de $35,5 \%$ e $11,3 \%$ respectivamente, para, pelo menos, um dos pais. A quantidade de sódio disponível para consumo nos domicílios brasileiros permanece duas vezes maior que o limite máximo de ingestão recomendado pela OMS. A disponibilidade excessiva de sódio ainda é observada em todas as regiões do país, nos meios urbanos e rurais e em todas as classes de renda (Sarno, Claro, Levy, Bandoni \& Monteiro, 2013). A prevalência do DM tem se elevado vertiginosamente em todo mundo, nas Américas o número de indivíduos com a doença foi projetado para 64 milhões para o ano de 2025. Nos países em desenvolvimento há uma tendência de aumento na frequência em todas as faixas etárias, especialmente nas mais jovens, cujo impacto negativo sobre a qualidade de vida e a carga da doença aos sistemas de saúde é imensurável (Sartorelli \& Franco, 2003). Dada a alta prevalência dos fatores de risco relacionados à história familiar, reforça-se ainda mais a importância da elaboração de ações que visem a reduzir a prevalência de fatores de risco exógenos.

O percentual de auto declaração de tabagismo apresentou-se em um percentual elevado $(7,14 \%)$, resultado preocupante, tendo em vista que o tabagismo é considerado pela OMS como a principal causa de morte previsível e um dos maiores problemas de saúde pública atualmente, sendo responsável por quase seis milhões de óbitos anuais no mundo e por perdas econômicas significativas (OMS, 2013).

O percentual de auto declaração de ingestão de bebida alcoólica apresentou-se igualmente elevado (67,14\%), resultado preocupante uma vez que o consumo de álcool também é prejudicial à saúde e pode originar diversos problemas sociais e econômicos, bem como acidentes automobilísticos e homicídios. As declarações dos estudantes entrevistados com relação ao frequente consumo de álcool vêm expor um importante problema de saúde pública. Resultados semelhantes em estudos realizados com população semelhante, fato que sugere que o consumo elevado álcool é facilitado por ser uma droga lícita e incentivada nas relações sociais. Assim como as outras drogas, o álcool leva a estado de consciência alterado e deixa o indivíduo susceptível a comportamentos de risco, como uso de outras drogas ilícitas (Jones, Oeltmann, Wilson, Brener \& Hill, 2001).

Um estudo realizado em uma universidade de Minas Gerais-MG no Brasil revelou como neste estudo um elevado consumo de álcool entre os universitários relacionando este consumo ao estado nutricional, tendo em vista que o consumo de bebidas alcoólicas pode influenciar na ocorrência de sobrepeso, obesidade e desnutrição naqueles que são consumidores moderados (Kachani, Brasiliano \& Hochgraf, 2008). Diante desse contexto é de suma importância a implementação de estratégias no sentido de diminuir a prevalência dessa conduta, na perspectiva de melhorar a qualidade de vida e prevenir o surgimento de comorbidades.

$O$ estilo de vida associado a fatores de risco tem expandido consideravelmente o desenvolvimento e agravamento das DCNT. A prática de atividade física (AF) regular surge como elemento relevante, pois atua na prevenção e terapia da obesidade e doenças associadas (Masson, Dias-da-Costa, Olinto, et al., 2005), contudo, a sociedade não alcança as recomendações mínimas para prática de AF (Hallal, Matsudo, Matsudo, et al., 2005), conforme recomendado pelo American College of Sports Medicine (Haskel, Lee, Pate, et al., 2007), para adultos saudáveis entre 18 e 65 anos, a prática de atividade física moderada a intensa deve ser por no mínimo 30 minutos em cinco dias da semana. Ainda relacionado ao estilo de vida, o presente estudo apresenta resultados semelhantes com o de Paixão, Dias e Prado

\footnotetext{
9 Trabalho Final do Curso de Especialização em Nutrição Humana - UNIFRA.

${ }^{10}$ Trabalho realizado com o intuito de descrever a proporção de fatores de risco para doenças cardiovasculares, dando ênfase aos fatores nutricionais, em alunos da área de saúde de uma universidade pública do Recife.
} 
(2010), que traz com maior predominância a inatividade física. Estudos realizados com adolescentes no Sul do Brasil mostram comportamentos de risco evidenciados em alunos de 18 a 19 anos, onde eram mais insuficientemente ativos que os seus pares mais jovens (15 a 17 anos) e os mais baixos níveis de AF foram encontrados entre as meninas (Farias, Nahas, Barros, et al., 2009), resultados similares com os achados neste estudo.

A alimentação dos estudantes, de maneira geral, foi considerada regular, apontando a existência de hábitos alimentares inadequados. Tal dado mostra a necessidade do aumento no consumo de frutas, legumes e verduras, além de a adequada ingestão hídrica, conforme recomendação da CGAN (2007). A vida universitária pode contribuir de diversas formas para práticas de vida não saudáveis. Uma delas diz respeito a deixar a casa dos pais e passar a viver em moradias estudantis, em casa de familiares ou dividindo moradia com amigos, que pode trazer importantes alterações na rotina diária, por conta das intensas tarefas acadêmicas e afazeres domésticos, pode haver falta de tempo para realizar refeições completas, bem como atividade física regular, além do estabelecimento de novos comportamentos e relações sociais (Marcondelli, Costa \& Schmitz, 2008).

Diante do exposto o RU visa auxiliar estudantes de baixa renda, oferecendo refeições com preço inferior ao mercado, chegando a ser gratuito para estudantes cadastrado em programas específicos de auxílio a estudantes de baixa renda e de cota. No caso do presente estudo, todos os estudantes entrevistados recebiam gratuidade para realizar suas refeições (Moura, et al., 2014).

Portanto é papel de um Serviço de Alimentação ligado à alimentação Universitária o oferecimento de alimentação equilibrada no contexto da assistência estudantil, como também é relevante que o Serviço desenvolva um importante papel de propagador de hábitos alimentares saudáveis e de conscientização da adoção de práticas alimentares que contribuam para a qualidade de vida e de saúde.

\section{Conclusão}

Os estudantes bolsistas frequentadores do RU que foram entrevistados, no geral, foram jovens com massa corporal adequada, maior parte insuficientemente ativo A e B e uma pequena parcela sedentários. Além disso, grande parte deles mostram condutas de risco para saúde, como o consumo de álcool, tabaco e hábitos alimentares inadequados. Foi observado também presença de sobrepeso e obesidade em uma parcela considerável, sendo que todos estes fatores são importantes na gênese de doenças crônicas como DM, HAS e DCV.

Neste sentido os restaurantes universitários entram com importante papel no cotidiano desses estudantes, uma vez que são espaços voltados para preparação e fornecimento de refeições que devem ser equilibradas em nutrientes e de fácil acesso para esses estudantes, por se localizarem dentro do campus da Universidade, privilegiando a comunidade acadêmica e sendo compatíveis com os horários de intervalos dos cursos. Desse modo é importante verificar a qualidade da alimentação oferecida pelo RU e adotar métodos para promoção de bons hábitos alimentares desses estudantes, por meio de ações de Educação Nutricional e Vigilância Nutricional, para isso se faz necessário o acompanhamento constante desse público.

\section{Referências}

ABESO - Diretrizes Brasileiras de Obesidade. (2016). [acesso Abr. 2017]. Retrieved from: <http://www.abeso.org.br/uploads/downloads/92/57fccc403e5da.pdf>.

ABESO - Mapa da Obesidade. (2010). [acesso em: 14 Abr. 2017]. Retrieved from: <http://www.abeso.org.br/atitudesaudavel/mapa-obesidade>.

Bandoni D. H., \& Jaime, P. C. (2008). A qualidade das refeições de empresas cadastradas no Programa de Alimentação do Trabalhador na cidade de São Paulo. Campinas, Rev. Nutr. Mar./Apr., 21 (2).

Batista, Filho M. (1999). Alimentação, Nutrição e Saúde. Rio de Janeiro: MEDSI.

Batista Filho, M., \& Rissin, A. (2003). A transição nutricional no Brasil: tendências regionais e temporais. Cad. Saúde Pública, Rio de Janeiro, 19(Sup. 1), S181-S191.

Brasil, Ministério da Saúde. (2014). Vigitel Brasil 2013: vigilância de fatores de risco e proteção para doenças crônicas por inquérito telefônico. Estimativas sobre frequência e distribuição sóciodemográfica de fatores de risco e proteção 
para doenças crônicas nas capitais dos 26 Estados brasileiros e no Distrito Federal em 2013. Brasília: Secretaria de Vigilância em Saúde, Secretaria de Gestão Estratégica e Participativa.

Coordenação Geral de Alimentação e Nutrição [Internet]. (2007). Como está sua alimentação? [acesso 2016 abril 11]. Retrieved from: <http://nutricao.saude.gov.br/teste_alimentacao.php>.

Costa, LCF, e FAG Vasconcelos. 2010. Influência de fatores socioeconômicos, comportamentais e nutricionais na insatisfação com a imagem corporal de universitárias em Florianópolis. SC. Rev. bras. epidemiol. 13 (4): 665-76. doi: 10.1590/S1415-790X2010000400011.

Durnin, J. V. G. A., \& Womersley, J. (1974). A gordura corporal avaliada a partir da densidade corporal total e sua estimativa de dobras cutâneas: medições em 481 homens e mulheres com idades de 16 a 72 anos (Body fat assessed from the total body density and its estimation from skinfold thickness: measurements on 481 men and women aged from 16 to 72 years). British Journal of Nutrition, (32) 77-97.

Farias, Jr J. C., Nahas, M. V., Barros, M.V. G., et al. (2009). Comportamento de risco à saúde em adolescentes no sul do Brasil: prevalência e fatores associados. Rev Panam Salud Publica, 25(4), 344-352.

Fernando, L. Z., Néstor, N. T., Julio, P. M., \& Ángel, R. A. (2001). Prevalencia de obesidad e hipercolesterolemia en la Facultad de Medicina de la Universidad Peruana Cayetano Heredia - 1998. Revista médica hered 12(3) 78-84.

Franca, C., \& Colares, V. (2008). Estudo comparativo de condutas de saúde entre universitários no início e no final do curso. Rev. Saúde Pública. 42(3), 420-7.

Garcia, R. W. D. (1993). Representações Sociais da Comida no meio urbano: Um estudo no centro da cidade de São Paulo. [Dissertação de Mestrado]. São Paulo. Faculdade de Saúde Pública da USP.

Guiesser, A. B. S., \&Lopes, L. C. C. (2013). Periódico do Instituto Brasileiro de Pesquisa e Ensino em Fisiologia do Exercício. Rev. Bras Nutr Espor, São Paulo, mai./jun., 39(7), 169-176.

Hallal, P. C., Matsudo, S. M., VKR Matsudo, V. K. R., et al. (2005). Physical activity in adults from two Brazilian areas: similarities and differences. Cad. Saúde Pública, Rio de Janeiro, mar-abr 21 (2): 573-580.

Haskel, W. L., I-Min L., Pate, R. R., et al. (2007). Physical Activity and Public Health: Updated Recommendation for Adults from the American College of Sports Medicine and the American Heart Association. Special reports, Official Journal of the American College of Sports Medicine, 1423-34.

INEP - Instituto Nacional de Pesquisa Anísio Teixeira. (2015). Censo da educação Superior 2013: Resumo técnico. Brasília: Instituto Nacional de Estudos e Pesquisas Educacionais Anísio Teixeira. Retrieved from: <http://download.inep.gov.br/download/superior/censo/2013/resumo_tecnico_censo_educacao_superior_2013.pdf $>$

Jones, S. E., Oeltmann, J., Wilson, T. W., Brener, N. D., \& Hill, C. V. (2001). Binge drinking among undergraduate college students in the United States: implications for other substance use. J Am Coll Health, 50(1), 33-8.

Kachani, A. T., Brasiliano, S., \& Hochgraf, P. B. (2008). O impacto do consumo alcoolico no ganho de peso. Rev Psiq Clin, 35(1), 21-24.

Lohman, T. G., Roche, A. F., \& Martorell, R. (1988). Anthropometric Standardisation Reference Manual. Champaign, IL: Human Kinetics Books.

Marcondelli, P., Costa, T. H. M., \& Schmitz, B. A. S. (2008). Nível de atividade física e hábitos alimentares de universitários do 3o ao 5ㅇsemestres da área da saúde. Rev. Nutr., Campinas, Feb., 21(1).

Masson, C. R., Dias-da-Costa, J. S., Olinto, M. T. A., et al. (2005). Prevalência de sedentarismo nas mulheres adultas da cidade de São Leopoldo, Rio Grande do Sul, Brasil. Cad. Saúde Pública, nov-dez, 21(6), 1685-1694.

Matsudo, S., Araújo, T., Matsudo, V., Andrade, D., Andrade, E., Oliveira, L. C., et al. (2001). Questionário Internacional de Atividade Física (IPAC): estudo de validade e reprodutividade no Brasil. Ver. Bras. Ativ. Saúde, 10:5-18.

Monteiro, C. A., Mondini, L., \& Costa, R. L. B. (2000). Mudanças na composição e adequação nutricional da dieta familiar nas áreas metropolitanas do Brasil (1988-1996). Rev Saude Publica, 34:3. 
Moura, MAP, et al. (2014). Grau de satisfação dos alunos da Universidade Federal do Piauí em relação aos serviços prestados pelo Restaurante Universitário do Campus Ministro Reis Velloso, em Parnaíba - PI. In: Congresso Internacional de Administração, Ponta Grossa Pr. Anais. Gestão Estratégica: Ética e Transparência nas Organizações.

OMS - Organização Mundial de Saúde. (2013). Informe OMS Sobre la epidemia mundial de Tabaquismo. Genova: WHO. [acesso em: 14 Abr. 2017]. Retrieved from: <http://apps.who.int/iris/bitstream/10665/85382/1/WHO_NMH_PND_13.2_spa.pdf?ua=1>.

OMS - Organização Mundial de Saúde. (2007). Obesity and overweight: What are overweight and obesity. [citado 2007 jul.] Retrieved from <http://www.who.int/mediacentre/factsheets/fs311/en/index.html>.

OMS - Organização Mundial de Saúde. (2002). Joint WHO/FAO Expert Consultation on Diet, Nutrition and the Prevention of Chronic Diseases. Geneva, Switzerland. Diet, nutrition and the prevention of chronic diseases: report of a joint WHO/FAO expert consultation, Geneva, 28 January -- 1 February 2002.

OMS - Organização Mundial de Saúde. (2000). Obesity: preventing and managing the global epidemic. Report of a World Health Organization Consultation. Geneva: World Health Organization. WHO Obesity Technical Report Series, (284), 256.

Paixão, L. A., Dias, R. M. R., Prado, W. L. (2010). Estilo de vida e estado nutricional de universitários ingressantes em cursos da área de saúde do recife/PE. Rev. Brasileira de Atividade Física \& Saúde. 15(3), 2010.

Petribú, MMV, Cabral, P. C., Arruda, I. K. G. (2009). Estado nutricional, consumo alimentar e risco cardiovascular: um estudo em universitários. Rev. Nutr., Campinas, nov./dez., 22(6), 837-846.

Peuker, A. C., Fogaça, J., \& Bizarro, L. (2006). Expectativas e beber problemático entre universitários. Psicologia: Teoria e Pesquisa, 22(2), 193-200.

Rezende, FAC, et al., (2006). Índice de Massa Corporal e Circunferência Abdominal: Associação com Fatores de Risco Cardiovascular. Universidade Federal de Viçosa, Viçosa, MG.

São Paulo (SP). (2010). Secretaria Municipal de Saúde. Coordenação de Epidemiologia e Informação - CElnfo. Boletim ISA - Capital 2008, no 2, 2011: Estado nutricional, insatisfação em relação ao peso atual e comportamento relacionado ao desejo de emagrecer na cidade de São Paulo. 2a edição. São Paulo: CElnfo.

Sarno, F., Claro, R. M., Levy, R.B., Bandoni, D. H., \& Monteiro, C. A. (2013). Estimativa de consumo de sódio pela população brasileira, 2008-2009. Revista de Saúde Pública. 47(3), 571-578.

Sartorelli, D. S., \& Franco, L. I. (2003). Tendências do diabetes mellitus no Brasil: o papel da transição nutricional. Artigo de Revisão. Cad. Saúde Pública, Rio de Janeiro, 19(Sup. 1), S29-S36.

Simão, C. B., Nahas, M. V., \& Oliveira, E. S. (2006). Atividade física habitual, hábitos alimentares e prevalência de sobrepeso e obesidade em universitários da universidade do planalto catarinense -UNIPLAC - Lages-SC. Revista Brasileira de Atividade Física \& Saúde. 11(1), 3-12.

Soar, C. Silva, O. S., \& Lira, J. G. (2012). Consumo alimentar e atividade física de estudantes universitários da área de saúde. Revista Univap. 18(31), 41-7.

Staudt, F. S., \& Mattos, K. M. (2011). Circunferência da Cintura e Risco Cardiovascular: Um estudo em Acadêmicos da Área de Saúde. Disc. Scientia. Série: Ciências da Saúde, Santa Maria, 12(1), 93-102. Recebido em: 05.04.2011. Aprovado em: 25.11.2011.

Tardio, A. P., \& Falcão, M. C. (2006). O impacto da modernização na transição nutricional e obesidade. Rev Bras Nutr Clin, 21(2), 117-24.

\section{Sobre os Autores}

Lisanah Rufino da Silva é estudante de graduação em nutrição pela Universidade Federal da Bahia - UFBA.

Maria da Conceição Pereira da Fonseca (PhD) é professora do Departamento de Ciências de Alimentos, da Escola de nutrição da Universidade Federal da Bahia, Doutora em Alimentos e Nutrição pela Universidade Estadual de Campinas - UNICAMP.

Carlos Rodrigo Nascimento de Lira é estudante de graduação em nutrição pela Universidade Federal da Bahia - UFBA. 
Bruno Santos de Assis é nutricionista do Núcleo de Segurança Alimentar da Pró-Reitoria de Administração da Universidade Federal da Bahia. Mestre em Alimentos e Nutrição pela Universidade Federal da Bahia - UFBA.

Celina Maria Pereira Alonso é professora do Departamento de Ciências de Alimentos, da Escola de Nutrição da Universidade Federal da Bahia, Mestre em Nutrição pela Universidade Federal da Bahia -UFBA. 